\title{
Alginate Microencapsulation of Human Islets Does Not Increase Susceptibility to Acute Hypoxia
}

\author{
I. K. Hals, ${ }^{1}$ A. M. Rokstad, ${ }^{1}$ B. L. Strand, ${ }^{1,2}$ J. Oberholzer, ${ }^{3}$ and V. Grill ${ }^{1,4}$ \\ ${ }^{1}$ Department of Cancer Research and Molecular Medicine, Faculty of Medicine, Norwegian University of \\ Science and Technology, Postbox 8905, 7491 Trondheim, Norway \\ ${ }^{2}$ Department of Biotechnology, Faculty of Natural Sciences and Technology, Norwegian University of \\ Science and Technology, 7034 Trondheim, Norway \\ ${ }^{3}$ Department of Surgery, University of Illinois, IL at Chicago, Chicago, IL 60612, USA \\ ${ }^{4}$ Department of Endocrinology, St. Olavs Hospital, Trondheim University Hospital, Postbox 3250, \\ 7006 Trondheim, Norway
}

Correspondence should be addressed to I. K. Hals; ingrid.hals@ntnu.no

Received 1 October 2013; Accepted 8 November 2013

Academic Editor: Norman Cameron

Copyright (C) 2013 I. K. Hals et al. This is an open access article distributed under the Creative Commons Attribution License, which permits unrestricted use, distribution, and reproduction in any medium, provided the original work is properly cited.

Islet transplantation in diabetes is hampered by the need of life-long immunosuppression. Encapsulation provides partial immunoprotection but could possibly limit oxygen supply, a factor that may enhance hypoxia-induced beta cell death in the early posttransplantation period. Here we tested susceptibility of alginate microencapsulated human islets to experimental hypoxia (0.1$0.3 \% \mathrm{O}_{2}$ for $8 \mathrm{~h}$, followed by reoxygenation) on viability and functional parameters. Hypoxia reduced viability as measured by MTT by $33.8 \pm 3.5 \%$ in encapsulated and $42.9 \pm 5.2 \%$ in nonencapsulated islets $(P<0.2)$. Nonencapsulated islets released $37.7 \%$ (median) more HMGB1 compared to encapsulated islets after hypoxic culture conditions $(P<0.001)$. Glucose-induced insulin release was marginally affected by hypoxia. Basal oxygen consumption was equally reduced in encapsulated and nonencapsulated islets, by $22.0 \pm 6.1 \%$ versus $24.8 \pm 5.7 \%$. Among 27 tested cytokines/chemokines, hypoxia increased the secretion of IL- 6 and IL-8/CXCL8 in both groups of islets, whereas an increase of MCP-1/CCL2 was seen only with nonencapsulated islets. Conclusion. Alginate microencapsulation of human islets does not increase susceptibility to acute hypoxia. This is a positive finding in relation to potential use of encapsulation for islet transplantation.

\section{Introduction}

Transplantation of pancreatic islets containing the insulinproducing beta cells could in principle cure type 1 diabetes. However, transplantation of allografts necessitates treatment with immunosuppressant drugs with ensuing side effects. Encapsulation of islets (or isolated beta cells) could potentially alleviate this problem, thus motivating previous and ongoing research on the feasibility of encapsulated islets for successful transplantation. Promising results (reversal of diabetes in animal models of diabetes) have been reported [14]. However, questions remain as to both the short and long term functionality of encapsulated islets or beta cells.

One question pertains to the impact of hypoxia on encapsulated islets. Native pancreatic beta cells have high rates of oxidative metabolism to meet the demand of insulin production and secretion [5], and even moderately decreased levels of oxygen have been shown to inhibit insulin release [6]. Hypoxia after transplantation is a major (albeit not the only) contributor to the dramatic drop of viable beta cells (nonencapsulated) that occurs in the immediate period following transplantation [7-10]. A negative impact of theinevitable-hypoxia during the immediate period following transplantation could possibly be worsened by encapsulation, since the distance of diffusion for oxygen could be greater in encapsulated versus nonencapsulated islets (or amassed beta cells) $[11,12]$, and a negative effect of clustering of islets may occur [13]. Comparisons of oxygen uptake in encapsulated versus nonencapsulated islets have been done for neonatal porcine [14] and pig [15] islets in vitro (normoxic conditions) 
without unveiling negative effects of encapsulation, while encapsulation of rat islets led to a significant reduction of oxygen uptake [16]. However, a similar comparison has, to the best of our knowledge, not been made for human islets, neither in a setting of normoxic nor hypoxic culture conditions.

The aim of the present study was to compare viability and functional parameters of encapsulated versus nonencapsulated islets during normoxic culture conditions and in particular after a defined period of hypoxia. We chose an in vitro approach, since testing could be influenced by site for transplantation, thereby modifying a basic impact of hypoxia. We used alginate microbeads, since such a preparation has recently been shown to be a promising candidate for immune protection in light of its low inflammatory potential $[17,18]$ as well as functional performance in mice models [1,2]. A recent study using similar microbeads highlights beneficial effects of encapsulation on human islet functionality [4].

\section{Materials}

Ultrapure sodium alginate from Laminaria hyperborea, Pronova UP-LVG (67\% guluronic acid, viscosity $1051 \mathrm{~mL} / \mathrm{g}$, endotoxin $23 \mathrm{EU} / \mathrm{g}$, MW $187 \times 10^{3}$ ) was from Nova-Matrix, Oslo, Norway. Other materials were from Sigma-Aldrich Chemicals Co. (St. Louis, MO) or from sources specified below.

\section{Methods}

3.1. Islet Procurement, Isolation, and Shipment. Human islets were isolated at the Division of Transplant, University of Illinois, Chicago, as previously described $[19,20]$ and shipped to Trondheim. Sustained function of shipped human islets has previously been documented [1]. The purity and viability (determined on the basis of dithizone staining) and insulin secretion at the time of shipment as well as donor characteristics are presented in Table 1 . Totally 5 shipments were received, each shipment containing islets from a single donor. The Regional Committee for Medical and Health Research Ethics Central, Norway, approved the procurement of human islets and their use for research. Only islets from donors with research consent were used.

3.2. Islet Culture and Microencapsulation. Upon arrival in Trondheim, islets were centrifuged (1000 rpm, $2 \mathrm{~min}$ ) and resuspended in RPMI 1640 medium containing $5.5 \mathrm{mM}$ glucose and supplemented with $10 \%$ fetal calf serum (FCS), $10 \mathrm{mM}$ HEPES, $4 \mathrm{mM}$ L-glutamine, $1 \mathrm{mM}$ sodium pyruvate, $100 \mathrm{IU} / \mathrm{mL}$ penicillin, and $100 \mu \mathrm{g} / \mathrm{mL}$ streptomycin. Islets were cultured in flasks at $37^{\circ} \mathrm{C}$ in a humidified atmosphere of $5 \% \mathrm{CO}_{2}$ in air.

The number of islets was estimated after overnight culture. One half of the islets was then encapsulated in alginate, while the other half remained nonencapsulated. For encapsulation, 10-20000 islet equivalents were centrifuged (1000 rpm, $2 \mathrm{~min}$ ) and resuspended in $300-400 \mu \mathrm{L}$ of RPMI with $5.5 \mathrm{mM}$ glucose. They were then added to $1.8 \mathrm{~mL}$ of
TABLE 1: Donor and donor islet characteristics.

\begin{tabular}{lcccc}
\hline Purity (\%) & Viability (\%) & $\begin{array}{c}\text { GSI prior to } \\
\text { shipment }\end{array}$ & Age (years) & BMI \\
\hline $87.3 \pm 2.7$ & $93.0 \pm 0.6$ & $2.1 \pm 0.4$ & $46.4 \pm 4.8$ & $30.2 \pm 2.7$ \\
$(80-95)$ & $(91-95)$ & $(1.24-3.22)$ & $(32-59)$ & $(22.6-40.8)$ \\
\hline
\end{tabular}

Data are mean \pm SEM (range), $n=5$.

GSI equals glucose stimulation index.

$2.0 \%$ sterile filtered UP-LVG alginate (in $0.3 \mathrm{M}$ mannitol, $\mathrm{pH}$ 7.3). Inhomogeneous alginate microbeads were formed by use of an electrostatic bead generator $(7 \mathrm{kV}$, one $0.4 \mathrm{~mm}$ needle, flow $10 \mathrm{~mL} / \mathrm{h}$ ) using a gelling solution of $50 \mathrm{mM} \mathrm{CaCl}_{2}, 1 \mathrm{mM}$ $\mathrm{BaCl}_{2}, 150 \mathrm{mM}$ mannitol, and $10 \mathrm{mM}$ HEPES, at $\mathrm{pH}$ 7.3. The microbeads (521 $\pm 10 \mu \mathrm{m}$ in diameter, measurements from 33 capsules, each containing 1-4 islets) were collected on a filter and then washed three times with $12 \mathrm{~mL}$ of Hank's and once with $20 \mathrm{~mL}$ of culture media before being transferred to a culture flask. Both encapsulated and nonencapsulated islets were cultured overnight and used for experiments 1-23 days later. We did not observe clumping of nonencapsulated islets to any major extent. Further, we did not detect obvious differences due to length of culture on function and viability neither in encapsulated nor in nonencapsulated islets.

3.3. Experimental Protocols. Prior to each experiment, aliquots from homogenous suspensions of encapsulated and nonencapsulated islets were collected to estimate the number of islets per $\mathrm{mL}$ culture media.

Encapsulated and nonencapsulated islets were divided into each of two groups and transferred to Petri dishes (with $<100$ islets per $5 \mathrm{~mL}$ culture medium) before exposure to either continuous normoxia or to $8 \mathrm{~h}$ of hypoxia followed by $14-18 \mathrm{~h}$ of reoxygenation. Hypoxia was induced by placing islets in a hypoxia chamber (Billups-Rothenberg Inc., Del Mar, CA) together with an oxygen monitor (Dräger Safety AG \& Co., KGaA, Lübeck, Germany) and a Petri dish with 5-10 mL of water for humidity. The chamber interior was flushed with nitrogen gas $\left(95 \% \mathrm{~N}_{2}, 5 \% \mathrm{CO}_{2}\right)$ until a level of $0.1 \% \mathrm{O}_{2}$ was reached. After 8 hours of incubation the oxygen concentration inside the chamber had risen to $0.2-0.3 \%$.

For each of the four different experimental conditions (i.e., normoxia or hypoxia for encapsulated and normoxia or hypoxia for nonencapsulated islets) a control estimate of islet number per dish was made also after the end of the reoxygenation period. Islets from the different culture conditions, as well as aliquots of culture media, were sampled for measurements as detailed below.

3.4. MTT. For each experimental condition, 30 or 40 islets (encapsulated as well as nonencapsulated) were handpicked into each of 2-5 parallel wells on a 24 -well plate for $4 \mathrm{~h}$ of exposure to 3-(4,5-dimethyl-2-thiazolyl)-2,5diphenyltetrazolium bromide (MTT). The MTT reagent in the media was removed by washing the islets several times in $0.9 \% \mathrm{NaCl}$. Islets were then incubated for one hour in $400 \mu \mathrm{L}$ DMSO per well at $37^{\circ} \mathrm{C}$ for color development. Fifty $\mu \mathrm{L} /$ well of $0.1 \mathrm{M} \mathrm{NaCl}$ in $0.1 \mathrm{M}$ Glycine, $\mathrm{pH} 10.5$, was added for color 
extraction. Two parallel aliquots per well were secured for absorbance measurements.

3.5. HMGB1. The amount of high mobility group box 1 (HMGB1) in media aliquots (kept at $-80^{\circ} \mathrm{C}$ until assay) was measured by a HMGB1 ELISA kit (IBL International, Hamburg, Germany). The assay was performed as recommended by the producer.

3.6. Insulin Secretion. Groups of 5 handpicked islets were placed in each of 5-6 parallel wells in a 24 -well plate. This was followed by preincubation for $30 \mathrm{~min}$ at $37^{\circ} \mathrm{C}$ in $0.5 \mathrm{~mL}$ of Krebs-Ringer bicarbonate buffer (KRB, containing $0.5 \%$ BSA and $10 \mathrm{mM}$ HEPES at pH 7.4) together with $1.6 \mathrm{mM}$ glucose. Islets were transferred into a new 24-well plate and incubated for another $60 \mathrm{~min}$ in $\mathrm{KRB}$ containing $1.6 \mathrm{mM}$ glucose in order to assess basal (= un-stimulated) insulin secretion. The same islets were finally transferred to a new 24 -well plate for stimulated insulin secretion by incubation for $90 \mathrm{~min}$ in KRB with $16.7 \mathrm{mM}$ glucose. Aliquots of incubation media were secured for basal as well as stimulated secretion. Samples were kept at $-20^{\circ} \mathrm{C}$ pending insulin measurements by a RIA kit for human insulin (Millipore, St. Charles, MO).

3.7. Oxygen Consumption. Equal amounts of islets were transferred to each of three dishes and exposed to hypoxia for $8 \mathrm{~h}$ followed by $14-18 \mathrm{~h}$ of reoxygenation. Control islets were cultured continuously at normoxia. For each condition islets (3x approx. 300 islets) were pooled into one sample immediately before the oxygen consumption measurements.

Oxygen consumption was measured by Clark-type polarographic oxygen sensors and high-resolution respirometry (Oxygraph-2k, OROBOROS, Innsbruck, Austria). Samples of up to 900 islets in culture medium (corresponding to $\sim 10^{6}$ islet cells $/ \mathrm{cm}^{3}$ ) were added to a chamber. Islets were allowed to sediment before closing the chamber, switching on magnetic stirring and recording oxygen uptake at basal respiration. The ATP synthase inhibitor oligomycin $(2 \mu \mathrm{g} / \mathrm{mL})$ was subsequently added with the aim to assess uncoupled (= not ATP-coupled) respiration. After that, the protonophore carbonyl cyanide p-trifluoromethoxyphenylhydrazone (FCCP) was added and titrated (up to $5-6 \mu \mathrm{M}$ ) to achieve a state of maximum respiratory capacity. Finally, rotenone $(0.5 \mu \mathrm{M})$ and antimycin $(2.5 \mu \mathrm{M})$, inhibitors of mitochondrial complexes I and III, were added in order to measure residual oxygen consumption (ROX). Oxygen consumption rates were calculated as the negative time derivate of the oxygen concentration present in the chamber ( $\mathrm{pmol} / \mathrm{s} / \mathrm{mill}$ cells). For all experiments, ROX values were close to zero.

3.8. Cytokine/Chemokine Measurements. For all islet culture conditions, aliquots of the culture media were harvested after the reoxygenation period. For a subset of experiments, aliquots of media were secured also immediately after the time of hypoxia exposure. Samples were kept at $-80^{\circ} \mathrm{C}$ until assays were performed.

Islet secreted mediators were analyzed by a multiplex bead-based cytokine assay (Bio-Plex Human Cytokine Group
I 10-Plex Panel, Bio-Rad Laboratories, Hercules, CA) containing the following analytics: IL-1ra, IL-6, IL-8/CXCL8, IL9, IL-10, IL-12(p70), GM-CFS, MCP-1/CCL2, MIP-1 $\beta / C C L 4$, and VEGF. In addition, we included Bio-Plex kit reagents for detecting MIF, a part of Human Cytokine Group II. Our panel of mediators was chosen on the basis of preanalyzed samples (from each of the four conditions) using a Bio-Plex Human Cytokine Group I 27-plex panel (BioRad Laboratories, Hercules, CA). The following analytics were below the detection limit and therefore excluded from the main analyses; IL-1 $\beta$, IL-2, IL-4, IL-5, IL-13, IL-15, IL17, Eotaxin/CCL11, Basic FGF, IFN- $\gamma$, IP-10/CXCL10, MIP$1 \alpha /$ CCL3, PDGF-BB, Rantes/CCL5, and TNF- $\alpha$. The multiplex analyses were performed as recommended by the producer except for using half the recommended amounts of beads. For intra-assay variations, see Supplementary Table S1 of the Supplementary Material available online at http://dx.doi.org/10.1155/2013/374925.

3.9. Microbead Dissolution, Extraction, and Measurement of DNA. To assess DNA contents microcapsules first had to be completely dissolved. Dissolving of alginate microbeads $(350 \mu \mathrm{L})$ was achieved by adding $1000 \mu \mathrm{L}$ of tetra sodium EDTA (50 mM, pH 8) at $37^{\circ} \mathrm{C}$ followed by intermittent vortexing for up to 30 minutes. After centrifugation (10 $\mathrm{min}$, $13000 \mathrm{rpm}$ ), the islet pellet was harvested and resuspended in water for extraction of DNA. Nonencapsulated islets were extracted by the same procedure. DNA was quantified by the Fluorescent DNA Quantification kit (Bio-Rad, Hercules, CA).

\section{Statistics}

Data are presented as mean \pm SEM. Also medians were calculated. The Wilcoxon rank test was used for significance testing. A $P$ value $<0.05$ was defined as statistically significant.

\section{Results}

5.1. MTT. Islet viability assessed by MTT is presented in Table 2. The mean absorbance values were identical for encapsulated and nonencapsulated islets after continuous normoxia. Previous hypoxia exposure significantly reduced the MTT parameter of viability in both groups of islets by $33.8 \pm 3.5 \%$ versus $42.9 \pm 5.2 \%$ ( $P<0.2$ for difference). There was thus no tendency for a stronger effect of hypoxia in the encapsulated islets.

5.2. HMGB1. Hypoxia-induced islet damage has been associated with HMGB1 release [21, 22]. The release of HMGB1 was therefore used as a marker for islet destruction. Compared to encapsulated islets, nonencapsulated islets released $22.4 \pm$ $13.3 \%$ more $(P<0.2)$ HMGB1 under continuous normoxia. Levels of HMGB1 were significantly increased in media from both groups of islets after experimental hypoxia by $37.2 \pm$ 15.2\% (median: $35.0 \%$ ) for encapsulated and by $39.7 \pm 28.7 \%$ (median: 33.3\%) for nonencapsulated islets $(P<0.2$ for 
TABLE 2: Absorbance values $(570 \mathrm{~nm})$ representing islet viability measured by MTT.

\begin{tabular}{lccccc}
\hline & \multicolumn{2}{c}{ Encapsulated islets } & & \multicolumn{2}{c}{ Non-encapsulated islets } \\
Normoxia & Hypoxia & Reduction by hypoxia (\%) & Normoxia & Hypoxia & Reduction by hypoxia (\%) \\
\hline $0.15 \pm 0.02$ & $0.10 \pm 0.01^{*}$ & $33.8 \pm 3.50$ & $0.15 \pm 0.02$ & $0.09 \pm 0.02^{*}$ & $42.9 \pm 5.20$ \\
\hline
\end{tabular}

${ }^{*} P<0.002$ for the effect of hypoxia, $P<0.2$ for the comparison of hypoxia-induced reduction of viability for encapsulated versus non-encapsulated islets. Data are mean \pm SEM for 12 separate experiments (two-five parallels per condition), one-four experiments per donor (five donors).

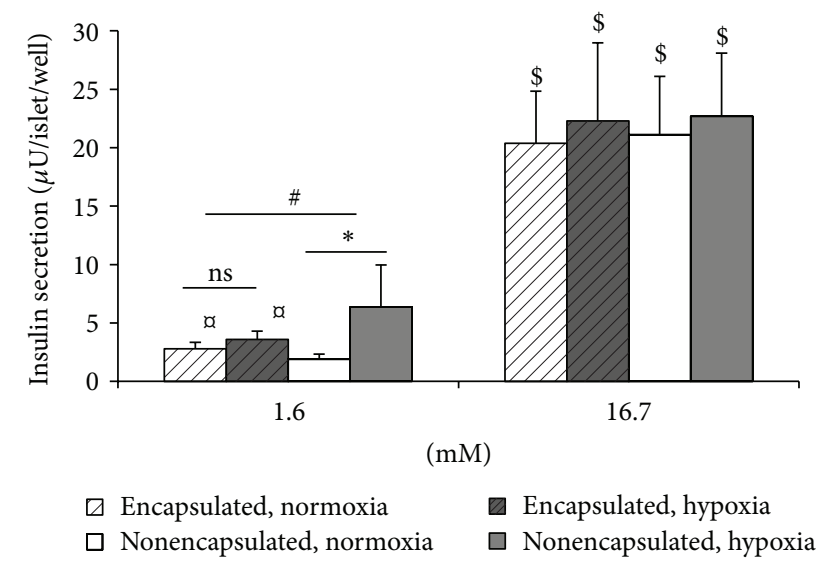

FIGURE 1: Effect of hypoxia and encapsulation on insulin secretion at 1.6 and $16.7 \mathrm{mM}$ glucose. ${ }^{\$} \mathrm{P}<0.02$ for the stimulatory effect of $16.7 \mathrm{mM}$ glucose, ${ }^{*} P<0.02$ for the effect of hypoxia, ${ }^{\mathrm{a}} P<0.04$ for the effect of encapsulation, and ${ }^{\#} P<0.02$ for the effect of hypoxia on encapsulated versus nonencapsulated islets at basal secretion. Data are mean \pm SEM of eight separate experiments (five-six parallels per experimental condition), one-three experiments per donor (four donors).

difference, $n=13)$. However nonencapsulated islets released in total $43.1 \pm 9.3 \%$ (median: $37.7 \%$ ) more HMGB1 than encapsulated islets under hypoxic culture conditions $(P<$ $0.001, n=13)$.

5.3. Insulin Secretion. Insulin release during normoxia and low glucose $(1.6 \mathrm{mM})$ was modest (in comparison to stimulated insulin release) for both encapsulated and nonencapsulated islets (Figure 1). Secretion in this unstimulated state was somewhat higher in encapsulated versus nonencapsulated islets conditions $(P<0.04)$. During the same conditions of oxygen supply (normoxia, no previous hypoxia) raising the glucose concentration to $16.7 \mathrm{mM}$ elicited a strong (1014-fold) insulin response in both types of islet preparations. The fold increase due to $16.7 \mathrm{mM}$ glucose, named the glucose stimulation index (GSI), was lower in encapsulated than in nonencapsulated islets $(10.0 \pm 3.1$ versus $15.9 \pm 4.7, P<$ 0.04). Interestingly, the GSI after shipment and culture in Trondheim for various times was higher than GSI after isolation, as recorded in Table 1.

After exposure to hypoxia, insulin secretion at low glucose concentrations increased in nonencapsulated islets $(P<0.04)$ but not in encapsulated islets (Figure 1). Hypoxia did not affect the stimulatory effect of $16.7 \mathrm{mM}$ glucose in absolute terms of insulin secretion whether in nonencapsulated or encapsulated islets. However, due to the increased insulin secretion during low glucose, the hypoxiainduced reduction of the GSI was more pronounced in nonencapsulated islets $(68.7 \pm 6.1 \%$, from $15.9 \pm 4.1$ to $3.9 \pm 0.9$, $P<0.02)$ than in encapsulated islets and $(24.5 \pm 11.2 \%$, from $10.0 \pm 3.1$ to $6.3 \pm 1.1, \mathrm{~ns})$.

5.4. Oxygen Consumption. The oxygen consumption in encapsulated and nonencapsulated islets following culture under normoxia or transient hypoxia is shown in Figure 2. At basal conditions after continuous normoxia oxygen consumption appeared slightly but not significantly higher in encapsulated versus nonencapsulated islets. Exposure to hypoxia reduced oxygen consumption both in encapsulated (by $22.0 \pm 6.1 \%$ ) and nonencapsulated islets (by $24.8 \pm 5.7 \%$, $P<0.7$ for comparison). Compared to basal respiration, addition of FCCP (an inducer of maximal respiration capacity) led to a modest increase in oxygen uptake for islets subjected to all four culture conditions. Hypoxia tended to reduce FCCP-induced respiration (by $9.7 \pm 13.1 \%$ in encapsulated and by $2.9 \pm 6.9 \%$ in nonencapsulated islets, $P<0.3$ for comparison) (Figure 2).

The lack of a "plateau" of oxygen uptake following the administration of oligomycin A (results not shown) hampered a more detailed analysis of the impact of hypoxia on oxygen consumption.

Islet DNA contents were measured in two representative experiments. Encapsulated islets contained a mean of $3.19 \mu \mathrm{g}$ DNA/sample and nonencapsulated islets $2.31 \mu \mathrm{g}$ DNA/sample. Corresponding values obtained after hypoxia were 2.63 and $2.12 \mu \mathrm{g}$ DNA/sample. These differences in DNA content parallel the slightly (ns) higher basal oxygen uptake seen in encapsulated versus nonencapsulated islets in Figure $2(+19.8 \pm 12.8 \%$ for normoxia and $+13.6 \pm 17.2 \%$ for hypoxia).

5.5. Cytokine/Chemokine Secretion. The most secreted mediators detected by the multiplex analysis were IL-6, IL8/CXCL8, MCP-1/CCL2, IL-9, IL12, and VEGF. The secretion profiles of these mediators from encapsulated and nonencapsulated islets after culture under normoxic and transient hypoxic conditions are given in Figure 3. The accumulation (pg or fg/islet/22-26h) of IL-8/CXCL8, IL-9, and MCP1/CCL2 was significantly increased from encapsulated versus nonencapsulated islets under basic normoxic conditions. The opposite tendency (reduced accumulation for encapsulated versus nonencapsulated islets) was seen for IL-12 (significant) and VEGF (nonsignificant). Experimental hypoxia significantly enhanced the accumulation of IL-6 and IL-8/CXCL8 


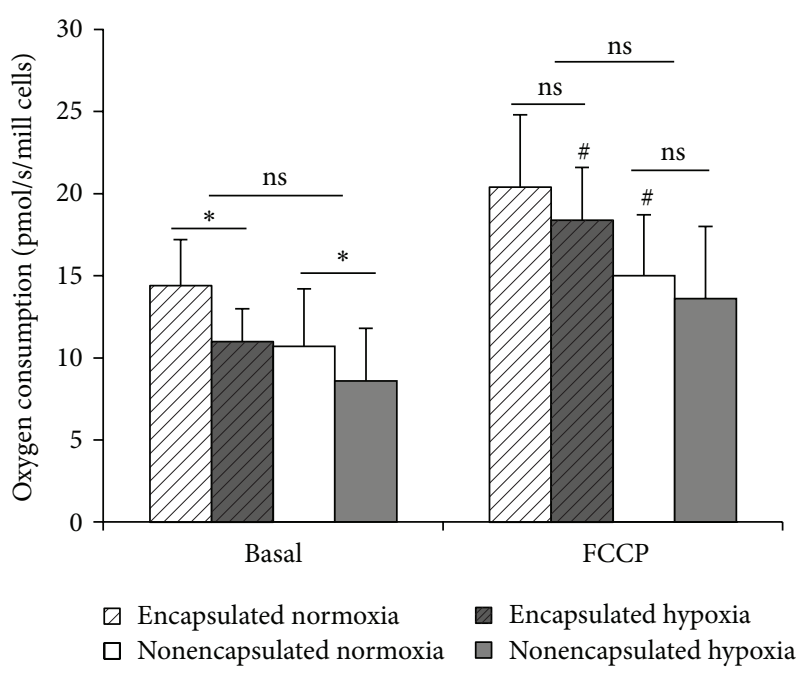

FigURE 2: Effect of hypoxia and encapsulation on oxygen consumption. ${ }^{*} P<0.05$ for the effect of hypoxia and ${ }^{\#} P<0.05$ for the effect of FCCP versus basal respiration. Data are mean \pm SEM of five-six separate experiments (one-three parallels per experimental condition), one-two experiments per donor (four donors).

for both groups of islets, whereas the secretion of MCP1/CCL2 was significantly increased from nonencapsulated while unchanged from encapsulated islets. Hypoxia did not affect the accumulation of IL-9 from neither group of islets. The secretion of IL-12 and VEGF was significantly reduced from nonencapsulated islets and unchanged for encapsulated islets.

The ratios of hypoxia-induced accumulation of cytokines/chemokines $(\mathrm{H})$ divided by the accumulation during continuous normoxia $(\mathrm{N})(\mathrm{H} / \mathrm{N}$ ratio) are summarized in Table 3. Notably, the hypoxia-induced enhancement or reduction of presented mediators was less pronounced for encapsulated islets versus nonencapsulated islets. Hence, the $\mathrm{H} / \mathrm{N}$ ratios were closer to unity for encapsulated islets.

Levels of IL-1ra, IL-10, GM-CSF, MIP-1 $\beta / C C L 4$, and MIF were close to the detection limit. These data are presented as Supplementary Figure S1 and Table S2. Significant effects of encapsulation were seen during basic conditions for MIP-1 $\beta / C C L 4$ and MIF (increased secretion) and IL-10 (decreased).

We also wished to address the question whether hypoxiainduced changes/alterations of the accumulation of cytokines take place mainly during the hypoxic event or following hypoxia (i.e., during reoxygenation). We compared in a subset of experiments, $n=4$ (representing two experiments for each of two donors/islet isolates), the accumulation during the $8 \mathrm{~h}$ of hypoxia with that which was secreted during the hypoxia + reoxygenation period. The data presented in Supplementary Table S3 show that the secretion of IL6, IL-8/CXCL8, MCP-1/CCL2, and VEGF continue to a large extent during the reoxygenation period. The most pronounced lingering effect of hypoxia was seen for IL-6. From nonencapsulated islets almost $90 \%$ was secreted during the time period of reoxygenation. Secretion differences between the hypoxia and the reoxygenation period tended to be less pronounced for encapsulated islets (Supplementary Table S3 and Supplementary Table S4). For IL-9 and IL-12 the secreted levels after $8 \mathrm{~h}$ of hypoxia were hardly detectable.

\section{Discussion}

Our results indicate, in general, that the impact of acute and severe hypoxia is no more negative for microencapsulated than for nonencapsulated human islets whether in terms of viability or function. In fact the effects of hypoxia on some parameters (MTT, HMGB1, and cytokines) were less, or tended to be less, marked in the encapsulated islets. The relevance of these findings would appear strengthened by the human islets being of high purity and displaying at the onset of experiments good function in terms of glucose-induced insulin secretion and oxygen consumption.

HMGB1 is secreted from human islets as a response to islet damage [21] and is recently shown to be secreted during hypoxia [22]. Our data confirm increased levels of HMGB1 secretion after hypoxic culture conditions, a finding shared by encapsulated and nonencapsulated human islets. However, we find less total secretion of HMGB1 from encapsulated versus nonencapsulated islets after hypoxia exposure. This suggests that the microcapsule could have a protective effect against islet destruction. As a further implication, one may note that HMGB1, due to its inflammatory properties of $[23,24]$, might be a contributor to the fibrotic responses against encapsulated islets, such responses being a major challenge in islet encapsulation based therapy. Also in this respect the lower secretion of HMGB1 from encapsulated islets could possibly be viewed as a positive finding. It should however be emphasized that the direct impact of HMGB1 in human islet transplantation has recently been questioned [21].

The insulin data point to subtle effects of encapsulation and in particular a slightly increased secretion during basal normoxic conditions. The cause(s) behind this difference has not been elucidated. Based on the hypoxiainduced rise in basal insulin release that we observe for nonencapsulated islets, one possible explanation could be that islets within the microcapsule are subjected to mild hypoxia, which subsequently gives rise to the basal secretion. Data from Vaithilingam et al. could support such notion since pretreatment with the hypoxia-mimicking desferrioxamine agent induces slightly elevated basal insulin secretion [25].

A high degree of oxygen consumption recorded in vitro has been linked to successful transplantations [26-28]. It is thus a positive finding (in terms of the clinical utility of encapsulated islets) that oxygen consumption under basic conditions (no hypoxia) was not diminished in encapsulated islets (Figure 2). These findings are in line with those reported previously for pig islets encapsulated in a monolayer cellular device [15] and for microencapsulated neonatal porcine islets [14]. The recorded values of oxygen flux ( $\mathrm{pmol} / \mathrm{s} / \mathrm{mill}$ cells) in our study are comparable to the findings for rat islets by 

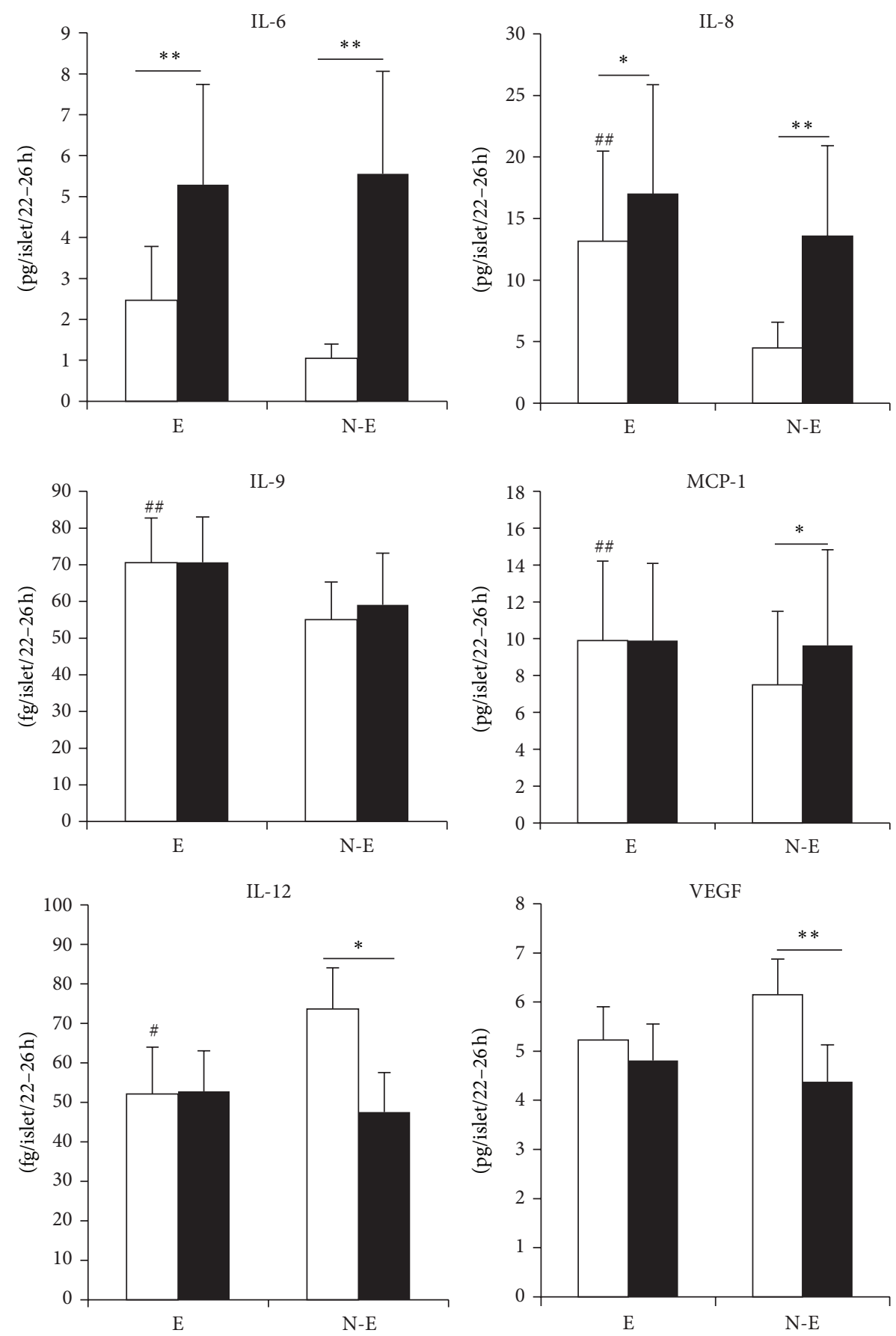

FIGURE 3: Secreted mediators from encapsulated (E) and nonencapsulated (N-E) islets following culture in continuous normoxia (open bars) and transient hypoxia (filled bars). ${ }^{*} P<0.03$ and ${ }^{* *} P<0.001$ for the effect of hypoxia, ${ }^{\#} P<0.05$ and ${ }^{\# \#} P<0.003$ for the effect of encapsulation during normoxia. Data are mean \pm SEM of 13 separate experiments (one sample per condition), one-five experiments per donor (five donors).

use of the same type of oxygraph [29]. As to the hypoxiainduced decrease in oxygen consumption that we find, this was anticipated from previous studies on nonencapsulated rat islets [30]. Importantly, the decrease in oxygen consumption due to hypoxia was not aggravated by microencapsulation.
The production and secretion of cytokines and chemokines are thought to reflect, in part at least, the impact of stressors on islets [31]. Cytokines and chemokines are of general importance in graft destruction due to their various roles in inflammation, angiogenesis, and microcapsule 
TABLE 3: Effect of hypoxia on islet secreted mediators by $\mathrm{H} / \mathrm{N}$ ratios (fold increase by hypoxia).

\begin{tabular}{lcccc}
\hline & \multicolumn{2}{c}{ Encapsulated islets } & \multicolumn{2}{c}{ Nonencapsulated islets } \\
& Mean \pm SEM & Median & Mean SEM & 4.66 \\
IL-6 & $3.52 \pm 0.80^{*}$ & 2.48 & $28.43 \pm 24.22^{* a}$ & \\
IL-9 & $0.97 \pm 0.11$ & 1.01 & $09 \pm 0.17$ & 0.95 \\
IL-12 & $0.88 \pm 0.07$ & 0.93 & $3.69 \pm 0.09^{* \#}$ & 0.71 \\
IL-8 & $1.59 \pm 0.23^{*}$ & 1.39 & $1.40 \pm 0.79^{* \#}$ & 2.11 \\
MCP-1 & $1.12 \pm 0.09$ & 0.98 & $0.75^{* \#}$ & 1.31 \\
VEGF & $0.91 \pm 0.06$ & 0.88 & $0.07^{* \#}$ & 0.72 \\
\hline
\end{tabular}

${ }^{*} P<0.001-0.05$ for the effect of hypoxia, ${ }^{\#} P<0.01-0.05$ for the comparison of encapsulated versus non-encapsulated islets. Data are based on 11-13 single experiments (one sample per condition), one-five experiments per donor (five donors). ${ }^{\mathrm{a}} \mathrm{Mean} \pm \mathrm{SEM}$ contains one outlier value. Excluding the outlier changes mean \pm SEM to $5.23 \pm 1.19$ and median to 4.14 .

$\mathrm{H} / \mathrm{N}$ equals ratio of hypoxic to normoxic conditions.

fibrosis. It was therefore of interest to compare the secretion of these biologically active substances from encapsulated and nonencapsulated islets.

Of the cytokines/chemokines that could be reliably measured, we found that IL-6, IL-8/CXCL8, IL-9, and MCP$1 /$ CCL2 were increased, or tended to be, by encapsulation, whereas IL-12 and VEGF tended to be decreased. IL-6 is generally known as a strong proinflammatory cytokine but is also shown to have anti-inflammatory properties $[32,33]$. Various regulatory roles of immune responses are also found for IL-9 [34] and IL-12 is an important regulator of helper T cells [35]. IL-8/CXCL8 is a strong attractor for neutrophils [36] and contributes to angiogenesis [37]. VEGF is a key proangiogenic growth factor $[38,39]$. MCP-1/CCL2 is an important chemokine in attraction of monocytes and has been negatively associated to graft function [40].

It should be noted that secretion of VEGF was decreased in response to hypoxia rather than increased. At first glance this finding in nonencapsulated islets is paradoxical, since treatment of human islets with the hypoxia-mimicking agent desferrioxamine increased the expression of VEGF [25]. However, a study with islets indicated that a $48 \mathrm{~h}$ culture in normoxia increased VEGF mRNA and protein to an extent that further increase was not observed after hypoxia [41]. Possibly, cells in the core of hypoxic islets have been damaged to an extent which could have impaired their production and secretion of VEGF. Also, our finding is in line with a previous paper showing reduced secretion of VEGF in response to a different stressor, that is, enterovirus infection [42]. It seems clear that the regulation of VEGF by different stressors under different experimental conditions needs to be studied further.

In general, the hypoxia-induced enhancement of cytokine/chemokine secretion appeared less marked from encapsulated islets. This could, in part at least, be secondary to the somewhat higher secretion during normoxic (no previous hypoxia) conditions. Whether this indicates that encapsulation induces more stress during basic conditions of culture cannot be decided. Speaking against such notion are the viability, oxygen consumption, and insulin data. In any case the data provided here do not indicate enhanced cytokine/chemokine responses to hypoxia in encapsulated versus nonencapsulated islets.
There are limitations to the study. With islets from a larger number of donors one could possibly have detected additional subtle differences between encapsulated and nonencapsulated islets. Further, one should acknowledge that we do not know which cells in the islet preparations that actually release the cytokines/chemokines. For IL-8/CXCL8 it has been shown that this cytokine can be produced by human $\beta$-cells [31]. Yet, clinically this uncertainty may be of less importance, since all cells that are part of an islet preparation will be transplanted. Also different encapsulation protocols have been used in previous studies, and we did not test for potential effects of such differences in the present context. However, the composition of alginate employed here has been widely used in recent years and transplantations of islets in similar microcapsules have given promising results in animal models of diabetes $[1,2,4]$, and future clinical studies with such capsules seem clinically relevant [4]. Finally, one cannot a priori extrapolate the present findings in vitro to a more complex in vivo situation. However, in a scenario in which one or several aspects of the transplantation of encapsulated islets are not successful the present data should be helpful in "problem-shooting" by minimizing the possibility of increased sensitivity to hypoxia.

\section{Conclusions}

This in vitro study has revealed subtle functional differences between alginate-encapsulated and free islets but none of these differences indicate that encapsulation increases susceptibility to negative effects of acute hypoxia. Indeed, the outcome of several parameters indicates better resilience towards hypoxia. This is a positive finding in relation to potential use of encapsulation for islet transplantation.

\section{Acknowledgments}

The authors are grateful to Kari Slørdahl for performing DNA quantifications and to Liv Ryan for performing multiplex analysis. I. K. Hals, A. M. Rokstad, and B. L. Strand are supported by Liaison Committee between the Central Norway Regional Health Authority (RHA) and the Norwegian 
University of Science and Technology (NTNU). J. Oberholzer is supported by the Chicago Project and NIH Grant R01 DK091526-01A1. V. Grill has received support from the Norwegian Diabetes Association.

\section{References}

[1] M. Qi, B. L. Strand, Y. Mørch et al., "Encapsulation of human islets in novel inhomogenous alginate- $\mathrm{Ca}^{2+} / \mathrm{Ba}^{2+}$ microbeads: in vitro and in vivo function," Artificial Cells, Blood Substitutes, and Immobilization Biotechnology, vol. 36, pp. 403-420, 2008.

[2] M. Qi, Y. Mørch, I. Lacik et al., "Survival of human islets in microbeads containing high guluronic acid alginate crosslinked with $\mathrm{Ca}^{2+}$ and $\mathrm{Ba}^{2+}$," Xenotransplantation, vol. 19, pp. 355-364, 2012.

[3] A. Remuzzi, R. Cornolti, R. Bianchi et al., "Regression of diabetic complications by islet transplantation in the rat," Diabetologia, vol. 52, no. 12, pp. 2653-2661, 2009.

[4] D. Jacobs-Tulleneers-Thevissen, M. Chintinne, Z. Ling et al., "Sustained function of alginate-encapsulated human islet cell implants in the peritoneal cavity of mice leading to a pilot study in a type 1 diabetic patient," Diabetologia, vol. 56, pp. 1605-1614, 2013.

[5] C. Hellerström, "Effects of carbohydrates on the oxygen consumption of isolated pancreatic islets of mice," Endocrinology, vol. 81, pp. 105-112, 1967.

[6] K. E. Dionne, C. K. Colton, and M. L. Yarmush, "Effect of hypoxia on insulin secretion by isolated rat and canine islets of Langerhans," Diabetes, vol. 42, no. 1, pp. 12-21, 1993.

[7] J. H. Juang, B. R. Hsu, C. H. Kuo, and S. W. Uengt, "Beneficial effects of hyperbaric oxygen therapy on islet transplantation," Cell Transplantation, vol. 11, no. 2, pp. 95-101, 2002.

[8] S. J. Hughes, S. E. Davies, S. H. Powis, and M. Press, "Hyperoxia improves the survival of intraportally transplanted syngeneic pancreatic islets," Transplantation, vol. 75, no. 12, pp. 1954-1959, 2003.

[9] N. Sakata, N. K. Chan, R. P. Ostrowski et al., "Hyperbaric oxygen therapy improves early posttransplant islet function," Pediatric Diabetes, vol. 11, no. 7, pp. 471-478, 2010.

[10] N. A. Deters, R. A. Stokes, and J. E. Gunton, "Islet transplantation: factors in short-term islet survival," Archivum Immunologiae et Therapiae Experimentalis, vol. 59, no. 6, pp. 421-429, 2011.

[11] J. Schrezenmeir, J. Kirchgessner, L. Gerö, L. A. Kunz, J. Beyer, and W. Mueller-Klieser, "Effect of microencapsulation on oxygen distribution in islets organs," Transplantation, vol. 57, no. 9, pp. 1308-1314, 1994.

[12] M. de Groot, T. A. Schuurs, and R. van Schilfgaarde, "Causes of limited survival of microencapsulated pancreatic islet grafts," Journal of Surgical Research, vol. 121, no. 1, pp. 141-150, 2004.

[13] A. S. Johnson, R. J. Fisher, G. C. Weir, and C. K. Colton, "Oxygen consumption and diffusion in assemblages of respiring spheres: performance enhancement of a bioartificial pancreas," Chemical Engineering Science, vol. 64, no. 22, pp. 4470-4487, 2009.

[14] J. P. Kitzmann, L. Law, A. Shome et al., "Real-time assessment of encapsulated neonatal porcine islet prior to clinical xenotransplantation," Xenotransplantation, vol. 19, pp. 333-336, 2012.

[15] S. Vériter, N. Aouassar, P.-Y. Adnet et al., "The impact of hyperglycemia and the presence of encapsulated islets on oxygenation within a bioartificial pancreas in the presence of mesenchymal stem cells in a diabetic Wistar rat model," Biomaterials, vol. 32, no. 26, pp. 5945-5956, 2011.
[16] A. S. Johnson, E. O’Sullivan, L. N. D’Aoust et al., "Quantitative assessment of islets of Langerhans encapsulated in alginate," Tissue Engineering, vol. 17, pp. 435-449, 2011.

[17] A. M. Rokstad, O. L. Brekke, B. Steinkjer et al., "Alginate microbeads are complement compatible, in contrast to polycation containing microcapsules, as revealed in a human whole blood model," Acta Biomaterialia, vol. 7, no. 6, pp. 2566-2578, 2011.

[18] A. M. Rokstad, B. I. Gustafsson, T. Espevik et al., "Microencapsulation of small intestinal neuroendocrine neoplasm cells for tumor model studies," Cancer Science, vol. 103, no. 7, pp. 12301237, 2012.

[19] M. Qi, B. Barbaro, S. Wang, Y. Wang, M. Hansen, and J. Oberholzer, "Human pancreatic islet isolation-part I: digestion and collection of pancreatic tissue," Journal of Visualized Experiments, no. 27, Article ID e1125, 2009.

[20] M. Qi, B. Barbaro, S. Wang, Y. Wang, M. Hansen, and J. Oberholzer, "Human pancreatic islet isolation-part II: purification and culture of human islets," Journal of Visualized Experiments, no. 27, Article ID e1343, 2009.

[21] R. Nano, L. Racanicchi, R. Melzi et al., "Human pancreatic islet preparation release HMGB1: (Ir)relevance for graft engrafment," Cell Transplant, vol. 22, no. 11, pp. 2175-2186, 2013.

[22] T. Itoh, M. Takita, J. A. SoRelle et al., "Correlation of released HMGB1 levels with the degree of islet damage in mice and humans and with the outcomes of islet transplantation in mice," Cell Transplant, vol. 21, pp. 1371-1381, 2012.

[23] M. T. Lotze and K. J. Tracey, "High-mobility group box 1 protein (HMGB1): nuclear weapon in the immune arsenal," Nature Reviews Immunology, vol. 5, no. 4, pp. 331-342, 2005.

[24] M. T. Lotze, H. J. Zeh, A. Rubartelli et al., "The grateful dead: damage-associated molecular pattern molecules and reduction/oxidation regulate immunity," Immunological Reviews, vol. 220, no. 1, pp. 60-81, 2007.

[25] V. Vaithilingam, J. Oberholzer, G. J. Guillemin, and B. E. Tuch, "Beneficial effects of desferrioxamine on encapsulated human islets-in vitro and in vivo study," The American Journal of Transplantation, vol. 10, no. 9, pp. 1961-1969, 2010.

[26] C. Fraker, M. R. Timmins, R. D. Guarino et al., "The use of the BD oxygen biosensor system to assess isolated human islets of Langerhans: oxygen consumption as a potential measure of islet potency," Cell Transplantation, vol. 15, no. 8-9, pp. 745-758, 2006.

[27] K. K. Papas, C. K. Colton, R. A. Nelson et al., "Human islet oxygen consumption rate and DNA measurements predict diabetes reversal in nude mice," The American Journal of Transplantation, vol. 7, no. 3, pp. 707-713, 2007.

[28] A. R. Pepper, C. P. Hasilo, C. W. J. Melling et al., “The islet size to oxygen consumption ratio reliably predicts reversal of diabetes posttransplant," Cell Transplant, vol. 21, pp. 2797-2804, 2012.

[29] K. Zacharovova, Z. Berkova, T. Spacek et al., "In vitro assessment of pancreatic islet vitality by oxymetry," Transplantation Proceedings, vol. 37, no. 8, pp. 3454-3456, 2005.

[30] E. S. O'Sullivan, A. S. Johnson, A. Omer et al., "Rat islet cell aggregates are superior to islets for transplantation in microcapsules," Diabetologia, vol. 53, no. 5, pp. 937-945, 2010.

[31] S. Negi, A. Jetha, R. Aikin, C. Hasilo, R. Sladek, and S. Paraskevas, "Analysis of beta-cell gene expression reveals inflammatory signaling and evidence of dedifferentiation following human islet isolation and culture," PLoS ONE, vol. 7, no. 1, Article ID e30415, 2012. 
[32] J. Scheller, A. Chalaris, D. Schmidt-Arras, and S. Rose-John, "The pro- and anti-inflammatory properties of the cytokine interleukin-6," Biochimica et Biophysica Acta, vol. 1813, no. 5, pp. 878-888, 2011.

[33] C. Garbers, H. M. Hermanns, F. Schaper et al., "Plasticity and cross-talk of interleukin 6-type cytokines," Cytokine and Growth Factor Reviews, vol. 23, pp. 85-97, 2012.

[34] R. J. Noelle and E. C. Nowak, "Cellular sources and immune functions of interleukin-9," Nature Reviews Immunology, vol. 10, no. 10, pp. 683-687, 2010.

[35] C. L. Langrish, B. S. McKenzie, N. J. Wilson, R. de Waal Malefyt, R. A. Kastelein, and D. J. Cua, "IL-12 and IL-23: master regulators of innate and adaptive immunity," Immunological Reviews, vol. 202, pp. 96-105, 2004.

[36] P. M. Murphy, "Neutrophil receptors for interleukin-8 and related CXC chemokines," Seminars in Hematology, vol. 34, no. 4, pp. 311-318, 1997.

[37] R. M. Strieter, M. D. Burdick, B. N. Gomperts, J. A. Belperio, and M. P. Keane, "CXC chemokines in angiogenesis," Cytokine and Growth Factor Reviews, vol. 16, no. 6, pp. 593-609, 2005.

[38] N. Ferrara and T. Davis-Smyth, "The biology of vascular endothelial growth factor," Endocrine Reviews, vol. 18, no. 1, pp. 4-25, 1997.

[39] N. Zhang, A. Richter, J. Suriawinata et al., "Elevated vascular endothelial growth factor production in islets improves islet graft vascularization," Diabetes, vol. 53, no. 4, pp. 963-970, 2004.

[40] R. Melzi, A. Mercalli, V. Sordi et al., "Role of CCL2/MCP-1 in islet transplantation," Cell Transplantation, vol. 19, no. 8, pp. 1031-1046, 2010.

[41] B. Vasir, L. P. Aiello, K.-H. Yoon, R. R. Quickel, S. Bonner-Weir, and G. C. Weir, "Hypoxia induces vascular endothelial growth factor gene and protein expression in cultured rat islet cells," Diabetes, vol. 47, no. 12, pp. 1894-1903, 1998.

[42] B. M. Schulte, K. H. Lanke, J. D. Piganelli et al., "Cytokine and chemokine production by human pancreatic islets upon enterovirus infection," Diabetes, vol. 61, pp. 2030-2036, 2012. 


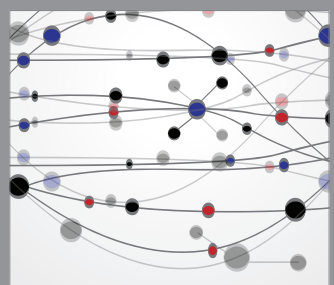

The Scientific World Journal
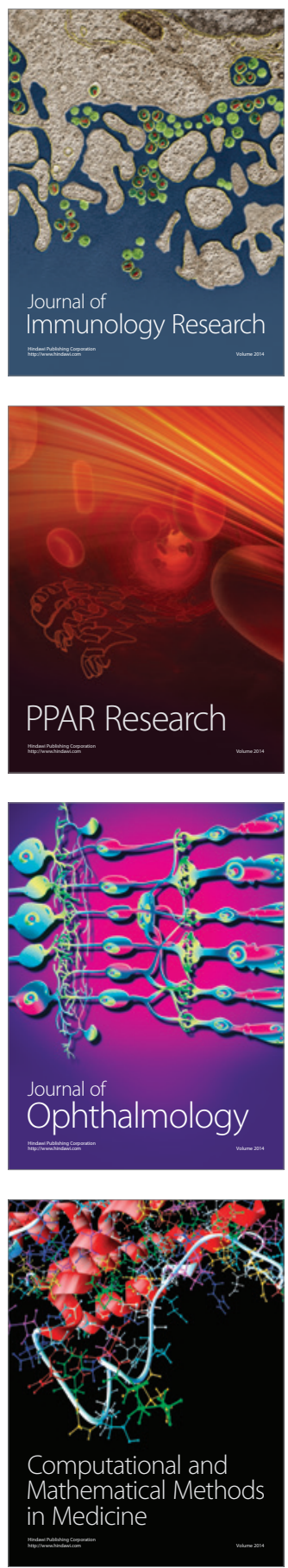

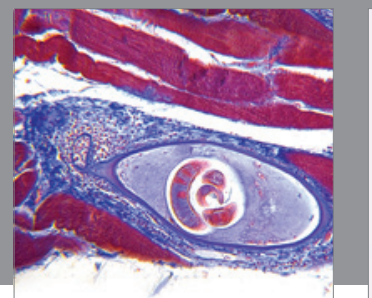

Gastroenterology

Research and Practice
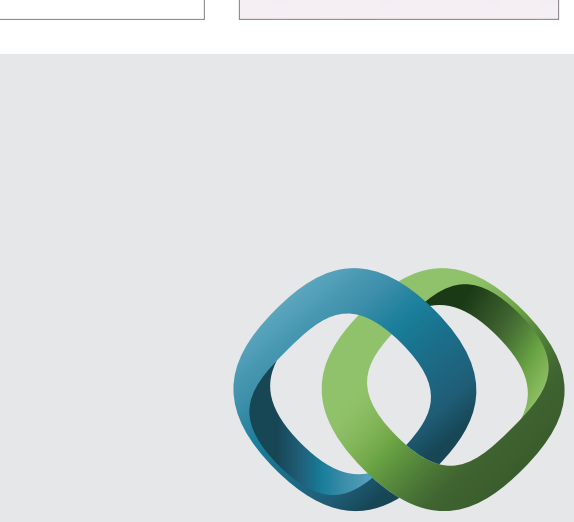

\section{Hindawi}

Submit your manuscripts at

http://www.hindawi.com
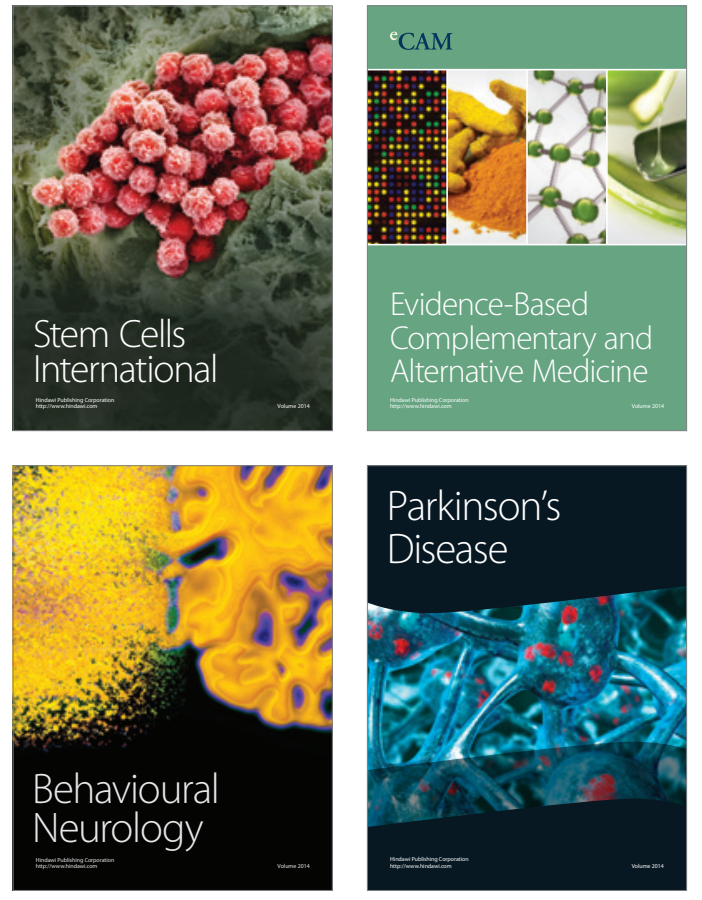
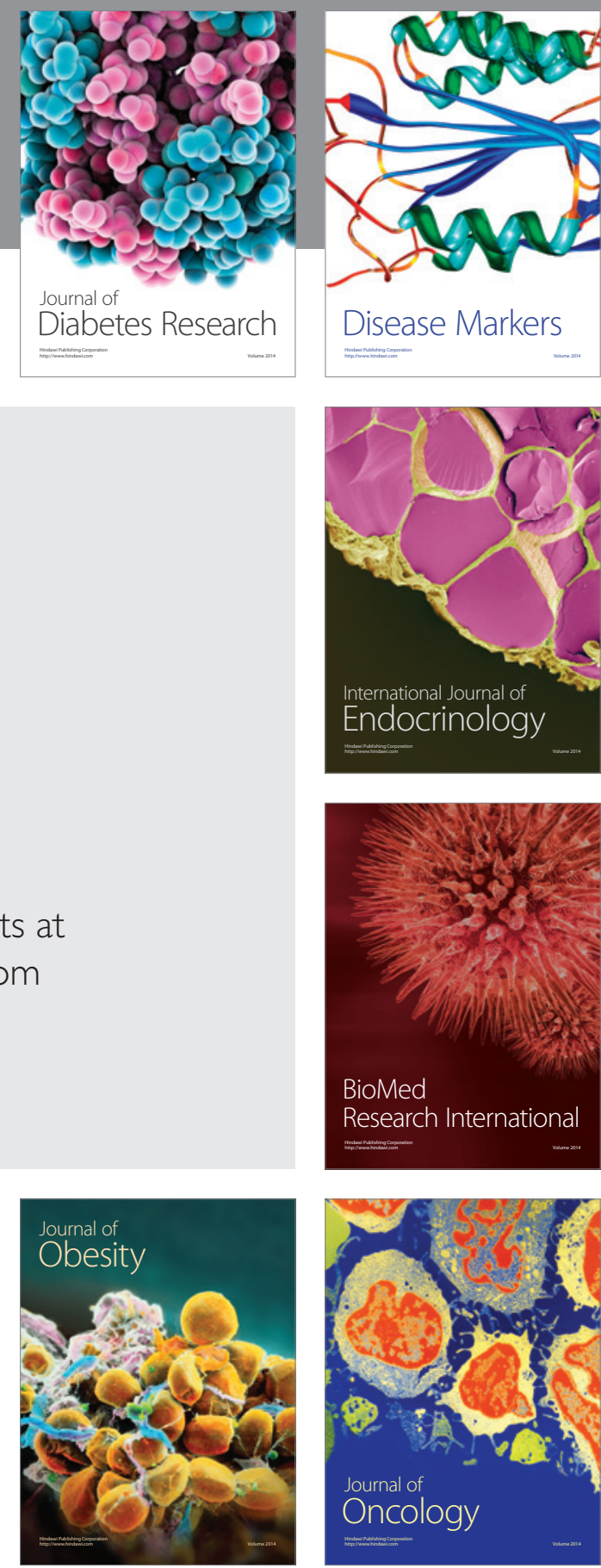

Disease Markers
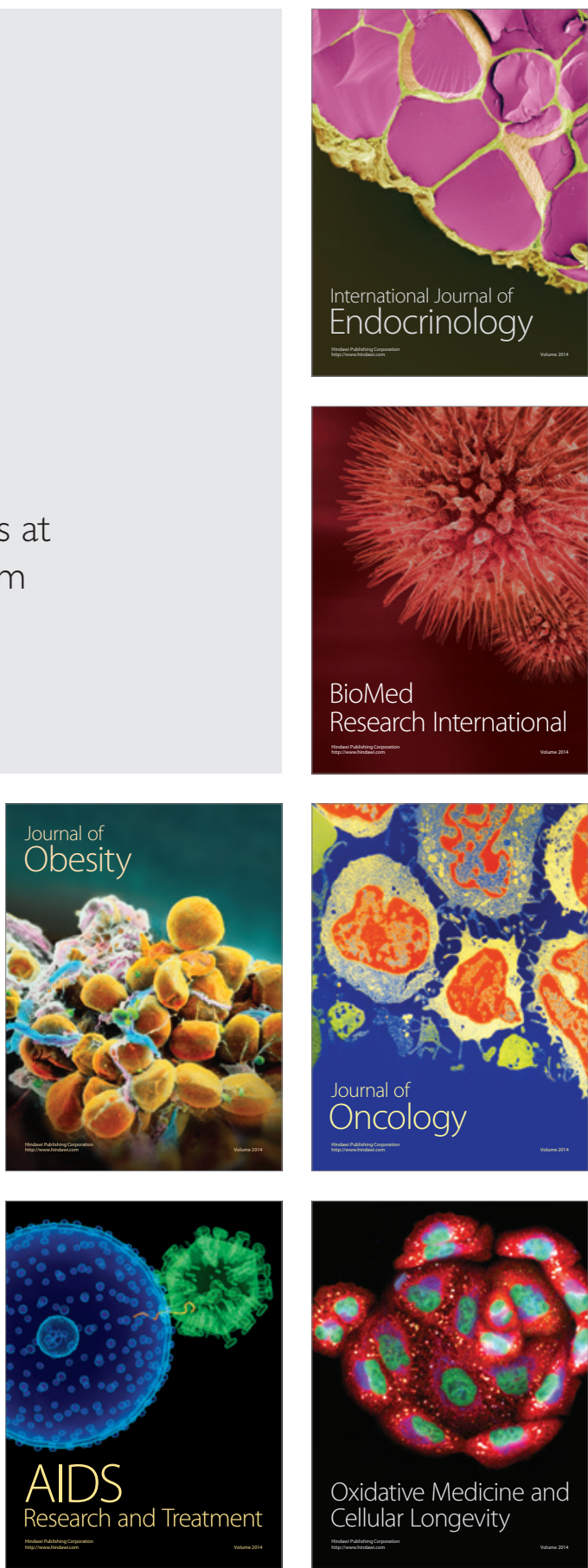